\title{
Nutritional risk and associated factors of adult in-patients at a teaching hospital in the Copperbelt province in Zambia; a hospital-based cross-sectional study
}

Nixon Miyoba ${ }^{1 *} \mathbb{D}$, Joseph Musowoya ${ }^{2}$,Emily Mwanza ${ }^{1}$, Angel Malama ${ }^{1}$, Nyati Murambiwa ${ }^{3}$, Irene Ogada ${ }^{4}$, Macriveness Njobvu ${ }^{1}$ and Doris Liswaniso ${ }^{5}$

\begin{abstract}
Background: Nutritional risk and undernutrition are common problems among medical and surgical patients. In hospital, malnutrition is frequently under-diagnosed and untreated thereby contributing to morbidity and mortality. The purpose of this study was to determine the prevalence of nutritional risk among adult inpatients at a teaching hospital in Zambia. In addition, the study sought to establish factors associated with nutritional risk.

Methods: A hospital-based cross-sectional study comprising of 186 consecutive in-patients aged 18-64 years admitted in medical and surgical wards was conducted at a teaching hospital. Out of one hundred and ninety eight (198) patients eligible to participate, complete data were collected from 186, representing a response rate of 93.9\%. The Malnutrition Universal Screening Tool was used to collect data over a period of six months. Evaluated patients were dichotomized into no risk and nutritional risk. Binary logistic regression was performed to identify variables associated with nutritional risk.

Results: The mean age of adult in-patients was $40.72 \pm 14.4$ years. Majority of the patients were male (61.8\%), while $38.2 \%$ were female. Results indicate that $59.7 \%$ of hospitalized patients were at nutritional risk. Vomiting, weakness, appetite decrease, dysphagia and weight loss were significantly associated ( $p=0.019, p=0.008, p<0.001, p=0.007$, and $p<0.001$ respectively) with nutritional risk. However, weight loss and appetite decrease were the most significant factors associated with nutritional risk $(\mathrm{OR}=50.16,95 \% \mathrm{Cl}=5.75-36.70, p<0.001$ and $\mathrm{OR}=28.06,95 \% \mathrm{Cl}=1.49-8.12$, $p=<0.001$ respectively).

Conclusion: Findings of our study suggest that close to $60 \%$ of adult inpatients at the teaching hospital were at nutritional risk. Nutritional risk is an issue of major concern at the teaching hospital and is associated with a number of variables. Identification of nutritional risk using Malnutrition Universal Screening Tool among adult inpatients is feasible in resource-poor settings like ours.
\end{abstract}

Keywords: Adult inpatients, Teaching hospital, Nutritional risk, Malnutrition, Undernutrition

\footnotetext{
* Correspondence: nixonmiyoba@gmail.com

${ }^{1}$ Kitwe Teaching Hospital, Kitwe, Zambia

Full list of author information is available at the end of the article
}

(c) The Author(s). 2018 Open Access This article is distributed under the terms of the Creative Commons Attribution 4.0 International License (http://creativecommons.org/licenses/by/4.0/), which permits unrestricted use, distribution, and reproduction in any medium, provided you give appropriate credit to the original author(s) and the source, provide a link to the Creative Commons license, and indicate if changes were made. The Creative Commons Public Domain Dedication waiver (http://creativecommons.org/publicdomain/zero/1.0/) applies to the data made available in this article, unless otherwise stated. 


\section{Background}

Nutritional risk is an important indicator used to predict the probability of clinical outcomes related to nutritional factors [1]. Upon admission to hospital, all patients should undergo routine nutritional risk screening [2]. Policy guidelines and protocols for identifying patients at nutritional risk should be in place in hospitals [1]. Screening is the initial step in identifying patients at nutritional risk $[1,2]$. It is a simple and rapid procedure used by health care professionals on first contact with patients [2]. Through screening, nutritional problems can be detected early and appropriate nutritional interventions developed for patients at significant risk [1].

Globally, a significant proportion (15-70\%) of hospitalized patients are undernourished $[3,4]$. In the United Kingdom, data from clinical settings indicates that 16$21 \%$ of patients are at nutritional risk $[5,6]$. The findings suggest that patients at medium and high risk of malnutrition experience frequent readmissions and stay longer in hospitals [5, 6]. A study conducted in Denmark reported that 14 out of 740 patients were at risk of malnutrition [1]. The prevalence of nutritional risk in a multicenter study conducted in Beijing Teaching Hospitals in China was estimated at $27.3 \%$ [7]. However, only $24.9 \%$ of the patients at nutritional risk received nutritional support [7].

Several studies have reported that undernutrition and nutritional risk are common problems among medical and surgical patients [8-11]. In hospital, malnutrition is frequently under-diagnosed and untreated thereby causing various adverse effects [12]. Malnutrition remains undiagnosed in close to $70 \%$ of hospitalized patients [3]. An estimated $80 \%$ of malnourished patients are discharged without receiving any nutritional support [3, 7]. Patients at nutritional risk have higher rates of infection, complications and suffer from organ failure [13]. Further, malnutrition results in delayed wound healing, suboptimal response to treatment, mortality and longer hospital stay [13-16]. Timely identification and management of malnutrition is required as the condition is not only a threat to a patients' health but also increases healthcare costs [17]. In order to mitigate the negative outcomes of malnutrition, the condition should be correctly identified and treatment of patients at nutritional risk prioritized [18].

In sub-Saharan Africa, there is limited literature about nutritional risk of hospitalized patients. In Zambia, there are no guidelines recommending a nutritional screening tool for use among adult in-patients. Further, there is paucity of literature in hospitals about the nutritional risk of in-patients making it difficult for health workers to prioritize treatment of patients at high risk. At the teaching hospital, there are no protocols for identification of in-patients at nutritional risk. It is against this background that this study has been conceptualized to provide baseline information on nutritional risk and associated factors among adult inpatients at the teaching hospital.

\section{Methods}

Research design, period and location

A hospital-based cross-sectional design was adopted for this study. The design was appropriate as data was collected from consecutive patients at one point in time [19]. The study was carried out from May to November 2017 at a teaching hospital, in Zambia. The teaching hospital has a bed capacity of 664 and is located in Kitwe District on the Copperbelt province [20]. The hospital offers tertiary health care services to the population of Kitwe, other towns on the Copperbelt province, and also provides referral services to patients from Luapula, Northern and North-Western provinces [20].

\section{Study population}

The study targeted adult in-patients (18-64 years). Adult in-patients admitted to medical and surgical wards and able to communicate were included in the study. The study excluded critically ill and cognitively impaired in-patients, those admitted in obstetric and gynaecology wards and those who declined to participate.

\section{Sampling techniques and sample size}

The teaching hospital was purposively sampled because it is the only teaching hospital offering tertiary health care services in Kitwe District [20, 21]. Medical and surgical wards were purposively sampled because nutritional risk is reported to be a significant problem among patients admitted in those wards [8-11]. Consecutive sampling was used to include all patients who met the inclusion criteria and consented to participate in the current study [7].

\section{Research instruments}

Identification of nutritional risk requires the use of validated and ease-to-use screening tools [22, 23]. In clinical settings, several nutrition screening tools are in use, each presenting variations in variability, reliability and acceptability $[24,25]$. There is no tool that is universally accepted for identifying nutritional risk [26]. However, reliability and validity are essential criteria when deciding on a screening tool to use [25]. Malnutrition Universal Screening Tool (MUST) used in similar studies was employed in data collection $[2,7,27]$. MUST was designed to identify patients at nutritional risk and is recommended for use among adult inpatients [2]. The tool has been found to be rapid, reproducible and internally consistent. There are three variables of the MUST: Body Mass Index (BMI), unintentional weight loss and disease effect [28]. Each variable is scored as 0,1 , or 2 and 
patients are classified into three categories of low risk (0), moderate risk (1) and high risk (2) [2]. Evaluated patients were dichotomized into no risk (low risk) and nutritional risk (moderate and high risk) [28]. Validity was ensured by use of an already validated questionnaire with elements of MUST incorporated into the questionnaire [27]. The tools were also pre-tested. During the pre-test, the test-retest method was used to establish reliability of the questionnaire. Data was collected twice at an interval of 3 days from 19 patients. A correlation coefficient of 0.79 (CI: 0.67-0.85) was computed from the two sets of data and found to be adequate [29] Additional file 1.

\section{Data collection procedures}

Names of admitted adult inpatients were obtained from admission registers in each ward. The investigator in consultation with sisters-in-charge of each ward generated a list of all eligible patients. Each patient was given a comprehensive explanation of the nature and purpose of the study. Voluntary informed consent was sought from participating patients prior to data collection.Guided by a structured questionnaire used in similar studies, face-to-face interviews were conducted in the local language with each patient on the ward [2, 27, 28]. Data on medical and demographic characteristics of patients was obtained from patient's files and recorded in the questionnaires. Additional information on other medical aspects such as diarrhoea, vomiting, dysphagia, appetite decrease, extent of weight loss and food intake was verbally solicited from patients and/or their bedsidders. Standard methods were employed by the investigator to measure weight and height [30]. The measurements were taken in the morning before meals. The weight of the patients was measured while wearing light clothing using the same clinical scale and recorded to the nearest $0.1 \mathrm{~kg}$. Scales were calibrated at the beginning of the study. Height was estimated to the nearest $0.1 \mathrm{~cm}$ using ulna length. Nutritional status data was enhanced by including information on mid-upper arm circumference and physical assessment [30]. At the end of each working day, MUST of all patients was computed using standard procedures and recorded by the investigators as: low risk (0), moderate risk (1) and high risk (2) [2]. Data was collected using the same procedure from consecutive patients for a period of six months (5/5/2017 to 10/11/2017).

\section{Statistical analyses}

Prior to data entry, completed questionnaires were checked daily for accuracy and consistency. Statistical Package for Social Sciences (SPSS version 21.0) was used to analyze data. Descriptive statistics in terms of means, frequencies, percentages and standard deviation were generated. Test of quantitative variables for normal distribution was done using the Kolmogorov-Smirnoff test, as appropriate. Binary multivariate logistic regression was performed to determine the influence of selected variables on nutritional risk. A $p$-value of less than 0.05 was considered statistically significant.

\section{Results}

Out of one hundred and ninety eight (198) patients eligible to participate, data was collected from $186(115$ male, 71 female) representing a response rate of $93.9 \%$. The mean age of the study population was $40.72(14.4)$ years (Table 1).

In this study, mid-upper arm circumference (MUAC) of all patients was evaluated. The mean MUAC of the patients was $24.01(4.92) \mathrm{cm}$, ranging from $14.5 \mathrm{~cm}$ to $41.2 \mathrm{~cm}$. The mean Body Mass Index of the patients was $22.65(2.4) \mathrm{Kg} / \mathrm{m}^{2}$. Patients' physical assessment revealed that $59(31.7 \%)$ were moderately wasted and 19(10.2\%) were severely wasted. Nutritional risk was determined by Malnutrition Universal Screening Tool (MUST) criteria. Results indicate that majority of the patients $83(44.6 \%)$ were at moderate risk, while $28(15.1 \%)$ were at high nutritional risk (Table 2).

As depicted in Table 2, evaluated patients were dichotomized into nutritional risk (moderate and high risk) and no risk (low risk). Results indicate that $59.7 \%$ of hospitalized patients were at nutritional risk.

Table 3 shows the characteristics/aspects associated with nutritional risk. The study reveals that weight loss and appetite decrease were the most significant factors associated with nutritional risk $(\mathrm{OR}=50.16,95 \% \mathrm{CI}=$

Table 1 Selected characteristics of adult in-patients

\begin{tabular}{llll}
\hline Participant characteristics & Categories & $\begin{array}{l}\mathrm{N}=186 \\
\mathrm{n}\end{array}$ & $\%$ \\
\hline Sex & Male & 115 & 61.8 \\
Readmission & Female & 71 & 38.2 \\
& Yes & 79 & 42.5 \\
Diarrhoea & No & 107 & 57.5 \\
& Yes & 20 & 10.8 \\
Vomiting & No & 166 & 89.2 \\
& Yes & 41 & 22.0 \\
Weakness & No & 145 & 78.0 \\
& Yes & 89 & 47.8 \\
Appetite decrease & No & 97 & 52.2 \\
& Yes & 96 & 51.6 \\
Dysphagia & No & 90 & 48.4 \\
Weight loss & Yes & 38 & 20.4 \\
& No & 148 & 79.6 \\
& Yes & 75 & 40.3 \\
& No & 111 & 59.7 \\
\hline
\end{tabular}


Table 2 Classification of nutritional risk based on MUST criteria and reorganized categories of nutritional risk is according to Velasco 2011 (Moderate and high risk = nutritional risk, low risk = no risk)

\begin{tabular}{lll}
\hline Nutritional risk classification & $\begin{array}{c}\mathrm{N}=186 \\
\mathrm{n}\end{array}$ & $\%$ \\
\hline Nutritional risk & & \\
$\quad$ Low risk & 75 & 40.3 \\
Moderate risk & 83 & 44.6 \\
High risk & 28 & 15.1 \\
Dichotomized nutritional risk & & \\
No risk & 75 & 40.3 \\
Nutritional risk & 111 & 59.7 \\
\hline
\end{tabular}

$5.75-36.70, p<0.001$ and $\mathrm{OR}=28.06,95 \% \mathrm{CI}=1.49-$ $8.12, p<0.001$ respectively).

\section{Discussion}

The findings of the present study reveal that $59.7 \%$ of adult in-patients were at nutritional risk. This result is consistent with that of a study conducted in Brazil which estimated the prevalence of nutritional risk at $60.7 \%$ [27]. In that study, data was collected using a structured questionnaire recommended by American Dietetic Association (ADA) [27]. A cross-sectional study in Danish hospitals indicated that close to $40 \%$ of patients were nutritionally at risk [31]. Another study conducted in three hospitals reported that $22.0 \%$ of inpatients were at nutritional risk [1]. Using Nutritional Risk Screening (NRS) 2002, a study in China estimated the prevalence of nutritional risk at $27.3 \%$ [7]. Results of a study in Britain using MUST as a screening tool reported a high prevalence of nutritional risk (19-65\%) across patient groups [32]. A similar study in Israel employing MUST to screen patients suggested that 33\% were at high nutritional risk [33].

The findings of our study are consistent with those reported in other clinical settings about the high prevalence of nutritional risk among adult inpatients [27, 31, 32].

Table 3 Characteristics associated with nutritional risk after binary logistic regression with OR (odds ratio) and 95\% confidence interval $(95 \% \mathrm{Cl})$

\begin{tabular}{llll}
\hline Characteristics & OR & $95 \% \mathrm{Cl}$ & $p$-value \\
\hline Sex & 0.65 & $(0.28-1.38)$ & 0.419 \\
Readmission & 1.36 & $(0.59-2.98)$ & 0.244 \\
Diarrhoea & 3.85 & $(0.46-2.98)$ & 0.050 \\
Vomiting & 5.55 & $(0.70-5.79)$ & 0.019 \\
Weakness & 7.07 & $(0.36-2.04)$ & 0.008 \\
Appetite decrease & 28.06 & $(1.49-8.12)$ & $<0.001$ \\
Dysphagia & 7.37 & $(0.42-4.05)$ & 0.007 \\
Weight loss & 50.16 & $(5.75-36.70)$ & $<0.001$ \\
\hline
\end{tabular}

Variations in prevalence of nutritional risk are attributed to the differing background characteristics in the patient population, pathology and screening tools applied [27]. For instance, the present study included different patients groups with various medical conditions admitted to medical and surgical wards. In addition, the study used MUST to determine nutritional risk among adult inpatients. However, MUST has been found to have excellent inter-rater reliability, concurrent and predictive validity $[32,34]$. The extent of nutritional risk in the current study might have been underestimated due to the exclusion of critically-ill and older patients from the study. Nutritional risk has been reported to be higher among older patients due to physiological changes, loss of appetite, functional decline and socioeconomic factors [35].

Nutritional risk of hospitalized patients is associated with several factors such as patient's health status, preadmission nutritional status and present pathology [27]. Other factors are related to physical, social and psychological dimensions of the patient. In order to mitigate the effects of malnutrition, it is important to identify nutritional risk based on predictive variables [27]. Identification of factors can facilitate attainment of patient's nutritional goals and aid in the formulation of appropriate interventions [36]. Studies have specifically identified weight loss, appetite decrease, vomiting and dysphagia as some of the potential factors associated with nutritional risk [27, 36, 37].

Several attempts have been made by investigators to establish potential factors associated with nutritional risk using various statistical models [38]. Similar to our study, previous studies have used binary logistic regression to determine predictive variables of nutritional risk [39, 40]. Variables of sex, weight loss, appetite decrease, vomiting, diarrhoea and dysphagia investigated in this study have also been reported in other studies [27, 36, 37]. The current study reveals that vomiting, weakness, appetite decrease, dysphagia and weight loss were significantly associated $(p<0.05)$ with nutritional risk. This finding is consistent with other studies that have reported a significant association between weight loss, appetite decrease, vomiting, dysphagia and nutritional risk [27, 36, 37].

The observation that weight loss was the most significant factor related to nutritional risk $(\mathrm{OR}=50.16,95 \% \mathrm{CI}$ $=5.75-36.70, p<0.001)$ was also reported in a study conducted in South America [27]. The study observed that weight loss was the strongest predictor of nutritional risk $(\mathrm{OR}=58.03$, 95\% CI: 18.46-182.41, $p<0.001)$ [27]. A similar study on nutritional risk among inpatients reported weight loss as the major predictor variable $(\mathrm{OR}=$ 37.7, 95\% CI: 10.7-57.3, $p<0.001$ ) [41]. Another study also reported that recent weight loss was associated with nutritional risk [1]. During hospitalization, many patients experience weight loss as a result of factors such as 
surgical procedures, disease effect and impaired appetite $[42,43]$. Among patients, the process of weight loss demonstrates caloric depletion [42].

A study on the prevalence of nutritional risk reported that appetite decrease was significantly associated with nutritional risk (OR $=10.31,95 \% \mathrm{CI}: 2.23-47.55, p=0.003$ ) [27]. Several studies have pointed out that appetite decrease increases the likelihood of nutritional risk and possesses the best sensitivity and specificity [36, 37, 41]. Consistent with other studies, appetite decrease in our study increased the chance of nutritional risk by approximately $28.06(\mathrm{OR}=28.06,95 \% \mathrm{CI}=1.49-8.12, p<0.001)$ $[37,41]$. Patient's appetite could be impaired as a result of the disease condition, treatment and psychological factors [44]. Reduced appetite contributes to weight loss and adversely affects a patient's nutritional status [27].

In the current study, vomiting and dysphagia were significantly associated with nutritional risk $(p=0.019$, and $p=0.007$ respectively). A similar study in Brazil found a significant association between vomiting, difficulties in swallowing and nutritional risk $(p<0.05)$ [27]. Studies suggest that variables associated with the digestive tract such as vomiting, dysphagia and diarrhoea are important risk factors $[43,45]$. In contrast to the finding that diarrhoea is significantly associated with nutritional risk $(p=0.025)$, the current study reported otherwise [27]. In our study, diarrhoea was not a significant predictor of nutritional risk $(p=050)$. This is a surprising finding considering that there is a growing recognition that diarrhoea undermines nutritional status [46]. The possible explanation is that the current study involved different patient groups in both medical and surgical wards. Some studies have suggested that nutritional risk is relatively higher among patients in medical wards [47].

Malnutrition "is a state in which imbalance of energy, protein and other nutrients causes measurable adverse effects on body form and functionality" [26, 30]. Among the adverse consequences of malnutrition is muscle wasting and weakness resulting in impairment of respiratory and cardiac functionality [42]. Body weakness related to skeletal muscles delays return to mobility among hospitalized patients [42]. This study asked patients on the degree of weakness and results indicate that the variable was significantly associated with nutritional risk $(\mathrm{OR}=7.07,95 \% \mathrm{CI}=0.36-2.04, p=0.008)$.

\section{Study limitations}

The current study collected data from one teaching hospital in Zambia, therefore, results may not be generalized to all hospitals in the country. In spite of the limitation above, this study has generated useful information on nutritional risk and related factors that may be appropriated at the teaching hospital. Further studies preferably longitudinal in nature maybe warranted to collect more information to allow for more rigorous analysis of results.

\section{Conclusions}

Findings of our study suggest that close to $60 \%$ of hospitalized patients at the teaching hospital are nutritionally at risk. Nutritional risk is an issue of major concern at the teaching hospital and is associated with a number of variables. Identification of nutritional risk using Malnutrition Universal Screening Tool among adult inpatients is feasible in resource-poor settings like ours. Weight loss and appetite decrease were the most significant factors associated with nutritional risk.

\section{Additional file}

Additional file 1: Questionnaire used in the study. (DOC $31 \mathrm{~kb}$ )

\section{Abbreviations}

ADA: American Dietetic Association; BMI: Body Mass Index; Cl: Confidence interval; $\mathrm{MoH}$ : Ministry of Health; MUAC: Mid-upper arm circumference; MUST: Malnutrition Universal Screening Tool; NRS: Nutritional Risk Screening; OR: Odds Ratio; SD: Standard deviation; SPSS: Statistical Package for Social Sciences; TDRC: Tropical Disease Research Center

\section{Acknowledgements}

The research was supported by management at the hospital.

\section{Funding}

The study was funded by the hospital management.

Availability of data and materials

Data will be available upon request from the corresponding author.

\section{Authors' contributions}

$\mathrm{NM}^{1}$ participated in study conceptualization, data collection, statistical analysis and drafting the manuscript. JM participated in critiqueing of study protocol and manuscipt. EM clarified concepts and edited the manuscript. AM clarified concepts, participated in statistical analysis and editing of the manuscript. $\mathrm{NM}^{2}$ clarified key concepts, edited the proposal and manuscript. IO read the proposal, clarified statistical analysis and edited the manuscript. MN edited the manuscript. DO edited the proposal and read the manuscript. All authors read and approved the final manuscript.

\section{Ethics approval and consent to participate}

Ethical clearance was obtained from Tropical Disease Research Center (TDRC) in Zambia. Permission and authority to conduct the study was obtained from management at the teaching hospital. Participants consented to participate in the study by providing their thump print on the consent form. Prior to data collection, they were briefed on the nature and objectives of the study. In order to assure anonymity and confidentiality, the names of the participants were not recorded in the questionnaires, codes were instead used as a form of identification. The filled questionnaires were kept in locked cupboards while the soft data files were password protected.

Consent for publication

Not applicable.

Competing interests

The authors declare that they have no competing interests. 


\section{Publisher's Note}

Springer Nature remains neutral with regard to jurisdictional claims in published maps and institutional affiliations.

\author{
Author details \\ ${ }^{1}$ Kitwe Teaching Hospital, Kitwe, Zambia. ${ }^{2}$ Ndola Teaching Hospital, Ndola, \\ Zambia. ${ }^{3}$ The University of Zambia, Lusaka, Zambia. ${ }^{4}$ Saint Francis Xavier \\ University, Antigonish, Canada. ${ }^{5}$ Ministry of Agriculture and Cooperatives, \\ Kalulushi, Zambia.
}

\section{Received: 3 February 2018 Accepted: 27 August 2018} Published online: 06 December 2018

\section{References}

1. Kondrup J, Johansen N, Plum LM, Bak L, Larsen IH, Martinsen A, et al. Incidence of nutritional risk and causes of inadequate nutritional care in hospitals. Clin Nutr. 2002;21:461-8.

2. BAPEN. The 'MUST' explanatory booklet. 2011. www.bapen.org.uk.

3. Lean M, Wiseman M. Malnutrition in hospitals. BMJ. 2008;336:290.

4. Barr J, Hecht M, Flavin KE, Khorana A, Gould MK. Outcomes in critically ill patients before and after the implementation of an evidence-based nutritional management protocol. Chest. 2004;125(4):1446-57.

5. Rust S, Cawood AL, Walters E, Stratton RJ, Elia M. Prevalence of malnutrition in hospital outpatients. Proc. nut. Soc. 2010;69(OCE2):E150.

6. Cawood AL, Rust S, Walter E, Stratton RJ, Elia M. The impact of malnutrition on health care use in hospital outpatients. Proc. nut. Soc. 2010;69(OCE2): E149.

7. Liang $X$, Jiang Z, Nolan MT, Wu X, Zhang H, Zheng Y, Liu H, Kondrup J. Nutritional risk, malnutrition (undernutrition), overweight, obesity and nutrition support among hospitalized patients in Beijing teaching hospitals. Asia Pac J Clin Nutr. 2009;18(1):54-62.

8. Fettes SB, Davidson HIM, Richardoson RA, Pennington CR. Nutritional status of elective gastrointestinal surgery patients pre- and post-operatively. Clin Nutr. 2002;21:249-51

9. Waitzberg DL, Caiaffa WT, Correia MITD. Hospital malnutrition: the Brazilian national survey (IBRANUTRI): a study of 4000 patients. Nutrition. 2001;17: 573-80.

10. Kruizenga HM, Wierdsma NJ, MAE VB, Schueren d v, Haollander HJ, JonkerSchuitema CF, et al. Screening of nutritional status in the Netherlands. Clin Nutr. 2003;22:147-52.

11. Gazzotti C, Albert A, Pepinster A, Petermans J. Clinical usefulness of the mini nutritional assessment (MNA) scale in geriatric medicine. J Nutr Health Aging. 2000:4:176-81.

12. Elia M, Russell CA. Combating malnutrition: recommendations for action: a report from the advisory group on malnutrition led by BAPEN, 2009.

13. American Society for Parenteral and Enteral Nutrition (ASPEN). Guidelines for the use of parenteral and enteral nutrition in adult and pediatric patients. JPEN. 2002;26(1 Suppl):1SA-9SA. 18SA-24SA

14. Prins. Nutritional assessment of the critically ill patients. S Afri J Clin Nutr. 2010;23(1):11-8

15. Wood C, Stubbs S, Warwick H, Dunnachie A, Elia M, Stratton R J. Malnutrition risk and health care utilisation in orthopaedic patients. Proc Nut Soc. 2004;63:(20A)1-8.

16. Stratton RJ, King CL, Stroud MA, Jackson AA, Elia M. 'Malnutrition universal screening tool' predicts mortality and length of hospital stay in acutely ill elderly. Br J Nutr. 2006:95(2):325-30

17. Brotherton A, Stroud M, Simmonds N. Malnutrition Matters: Meeting quality standards in nutrition care. BAPEN Quality Group, BAPEN. 2010.

18. Bowers S. Nutrition support for malnourished, acutely ill adults. MEDSURG Nurs. 1999;8:145-64

19. Katzenellenbogen JM, Joubert G, AbdoolKarim SS. Epidemiology. A manua for South Africa. Cape Town: Oxford University Pres; 2002.

20. Teaching hospital. Action plan. Kitwe: Teaching hospital; 2016.

21. McPake B, Nakamba P, Hanson K, McLoughlin B. Private wards in public hospitals: two-tier charging and the allocation of resources in tertiary hospitals in Zambia. Lusaka: Health Economics and Financing Programme; 2004

22. Vellas B, Villars H, Abellani G, Soto ME, Rolland Y, Guigoz Y, Morley JE, Chumlea W, Salva A, Rubenstein LZ, Garry P. Overview of the MNA (R) - its history and challenges. J Nutr Health Ageing. 2006;10:456-63.
23. Elia M, Stratton R, Russell C, Green C, Pang F. The cost of disease-related malnutrition in the UK and economic considerations for the use of oral nutritional supplements (ONS) in adults. In: Health Economic Report on Malnutrition in the UK. Redditch: BAPEN; 2005.

24. Green SM, Watson R. Nutritional screening and assessment tools for older adults: literature review. J Adv Nurs. 2006;54:477-90.

25. Holmes $\mathrm{S}$. The effects of undernutrition in hospitalized patients. Nurs Stand. 2007;22(12):35-8.

26. Green SM, Watson R. Nutritional screening and assessment tools for use by nurses: literature review. J Adv Nurs. 2005;50:69-83.

27. de Aquino PST. Identification of malnutrition risk factors in hospitalized patients. Rev Assoc Med Bras. 2011;57(6):623-9.

28. Velasco C, Garcia E, Rodriguez V, Frias L, Garriga R, Alvarez J, Garcia-Peris P, Leon M. Comparison of 4 nutritional screening tools to detect nutritional risk in hospitalized patients. A multicentre study. European journal of clinical nutrition. Nat Publ Group. 2010;65(2):1-23.

29. Murphy KR, Davidshofer CO. Psychological testing: principles and applications. 6th ed. Upper Saddle River: Pearson; 2005

30. Elia M. Screening for malnutrition: a multidisciplinary responsibility. Development and use of the. In: 'Malnutrition Universal Screening Tool' ('MUST') for adults. MAG, a Standing Committee of BAPEN. Redditch: BAPEN; 2003.

31. Rasmussen HH, Kondrup J, Staun M, Ladefoged K, Kristensen H, Wengler A. Prevalence of patients at nutritional risk in Danish hospitals. Clin Nutr. 2004; 21:1009-15

32. Stratton RJ, Hackston A, Longmore D, Dixon R, Price S, Stroud M, King C, Elia M. Malnutrition in hospital outpatients and inpatients: prevalence, concurrent validity and ease of use of the 'malnutrition universal screening tool' ('MUST') for adults. Br J Nutr. 2004;92:799-808.

33. Ben-Ishay O, Gertsenzon H, Mashiach T, Kluger Y, Chermesh I. Malnutrition in surgical wards: a Plea for concern. Gastroenterol Res Pract. 2011;2011:1-4.

34. Kondrup J, Rasmussen HH, Hamberg O. Nutrition risk screening (NRS 2002): a new method based on an analysis of controlled clinical. Clin Nutr. 2003: 22:321-36.

35. Sullivan DH, Sun S, Walls RC. Protein-energy undernutrition among elderly hospitalized patients: a prospective study. J Am Diet Assoc. 1999;281:2013-9.

36. Detsky AS, McLaughlin JR, Baker JP, Johnston N, Whittajer S, Mendelson R, et al. What is subjective global assessment of nutritional status? JPEN J Parenter Enteral Nutr. 1987:11:8-13.

37. Anthony PS. Nutritional screening tool for hospitalized patients. Nutr Clin Pract. 2008:23:373-82

38. Talukder A, Factors associated with malnutrition among under-five children: illustration using Bangladesh demographic and health survey, 2014

39. Das S, Gulshan J. Different forms of malnutrition among under five children in Bangladesh: a cross sectional study on prevalence and determinants. BMC Nutr. 2017:3:(1)2-12.

40. Das $\mathrm{S}$, Hossain MZ, Islam MA. Predictors of child chronic malnutrition in Bangladesh. Proc Pak Acad Sci. 2008:45:137-55.

41. Kruizenga HM, Seidell JC, Vet HCW, Wierdsma NJ. Van Bokhorstde van der Schueren MAE. Development and validation of a hospital screening tool for malnutrition: the short nutritional assessment questionnaire. Clin Nutr. 2005; 24:75-82.

42. Schenker S. Undernutrition in the UK. Br Nutr Found Nutr Bull. 2003;28:87-120.

43. Ferguson M, Capra S, Bauer J, Banks M. Development of a valid malnutrition screening tool for adult acute hospital patients. Nutrition. 1999:15:458-64.

44. Muscaritoli M, Aversa Z, Laviano A, Fanelli FR, Anker SD, Argilés J, Bauer JM, Sieber CC, Biolo G, Boirie Y, Bosaeus I, Cederholm T, Costelli P, Fearon KC, Maggio M, Schneider SM, 22 Schols A. Consensus definition of sarcopenia cachexia and pre-cachexia: joint document elaborated by special interest groups (SIG) " cachexia-anorexia in chronic wasting diseases" and " nutrition in geriatrics". Clin Nutr. 2010;29:154-9.

45. Ward J, Closet J, Little J, Boorman J, Perkins A, Coles SJ, et al. Development of a screening tool for assessing risk of undernutrition in patients in the community. J Hum Nutr Diet. 1998;11:323-30.

46. Brown KH. Diarrhoea and malnutrition. J Nutr. 2003;133(1):328S-32S.

47. Giryes S, Leibovitz E, Matas Z, Fridman S, Gavish D, Shalev B, Ziv-Nir Z, Berlovitz $Y$, Boaz M. Measuring nutrition risk in hospitalized patients. IMAJ. 2012;14:2-6. 